\title{
Promising Poly $(\varepsilon$-caprolactone) Composite Reinforced with Weft-Knitted Polyester for Small-Diameter Vascular Graft Application
}

\author{
Fu-Jun Wang, Abedalwafa Mohammed, Chao-Jing Li, and Lu Wang \\ Key Laboratory of Textile Science and Technology, Ministry of Education, College of Textiles, Donghua University, \\ Shanghai 210620, China \\ Correspondence should be addressed to Lu Wang; wangfujun@dhu.edu.cn
}

Received 25 March 2014; Revised 7 June 2014; Accepted 16 June 2014; Published 9 July 2014

Academic Editor: Joong-Hee Lee

Copyright (C) 2014 Fu-Jun Wang et al. This is an open access article distributed under the Creative Commons Attribution License, which permits unrestricted use, distribution, and reproduction in any medium, provided the original work is properly cited.

\begin{abstract}
The present study was designed to improve the mechanical performance of a small-diameter vascular prosthesis made from a flexible membrane of poly( $\varepsilon$-caprolactone) (PCL). PCL reinforcement was achieved by embedding a tubular fabric knitted from polyethylene terephthalate (PET) yarns within the freeze-dried composite structure. The knitting density of PET fabric influenced the mechanical properties of the new vascular graft. Results showed that the composite prototype has good mechanical properties, water permeability, elastic recovery, and suture retention strength. Increases in loop density increased compressive strength and suture retention strength and decreased elastic recovery. The new composite prototype vascular graft has promising potential applications in clinics because of its excellent mechanical properties.
\end{abstract}

\section{Introduction}

Atherosclerotic vascular disease is one of the most common causes of death and disability worldwide [1]. Vascular grafts, which are commercially available, are special tubes that serve as artificial replacements for damaged and diseased blood vessels; these grafts are mainly produced from polyesterknitted or woven fabrics $[2,3]$ or expanded polytetrafluoroethylene (ePTFE) [4-7]. Polyethylene terephthalate (PET) is well recognised in biomedical applications because of its biocompatibility, resilience, flexibility, durability, and resistance to biodegradation and sterilisation. Whilst PET has been successfully used in large-diameter grafts, smallcaliber grafts still show unacceptably high percentages of failure. Blood plasma proteins are absorbed by the graft because of surface forces, eventually resulting in inflammation, infection, thrombus formation, and vessel reclosure [8]. These events may cause thrombosis and induce progressive degradation in permeability, especially in terms of vascular permeability, because of the hemodynamic conditions and mechanical inadequacies of the implants. The major cause of the poor performance of such prostheses is lack of compliance [9]. Thus, utilising vascular prostheses from a biocompatible material with the same structural and mechanical properties as the vascular tissue is ideal $[10,11]$. Poly( $\varepsilon$-caprolactone) (PCL) is a promising biodegradable polymer with a long degradation time that has been widely used both in vivo and in vitro [12-16]. PCL small-diameter grafts are promising alternatives to ePTFE grafts because the healing characteristics of the former are better than those of the latter [17]; PCL also presents excellent characteristics, such as biodegradability, biocompatibility, flexibility, and ease of manufacture $[17,18]$. PCL has been used as a raw material to produce small-diameter blood vessel scaffolds by electrospinning. However, the mechanical properties of this material cannot comply with vascular graft requirements $[19$, 20]. Textile fabric parameters, such as fabric design and yarn properties, influence the properties of vascular prostheses (porosity, permeability, and mechanical performances) [21, 22]. In this study, tubular PCL reinforced with different PET-knitted fabric structures to form multilayer composites is considered as a novel biomedical material for preparing vascular grafts. The material imitates human blood vessels, which are composed of three layers. PET-knitted fabric 
was used to increase the mechanical properties of the PCL prototype vascular graft. The influence of fabric density and yarn properties on the mechanical properties of the PCL/PET composite prototype vascular graft was also investigated.

\section{Materials and Methods}

2.1. Materials. PET monofilaments and multifilaments (30D) were purchased from Shaoxing Fangxin Chemical Fibre Company (Zhejiang, China) and used for the weft-knitted tubular fabric. The properties of PET monofilaments and multifilaments are shown in Table 1. PCL was purchased from Shenzhen Bright China Industrial Co., Ltd. (Shenzhen, China). Acetic acid was purchased from Sinopharm Chemical Reagent Co., Ltd. (Shanghai, China), and used as a solvent.

2.2. Methods. A PCL tubular freeze-dried graft was fabricated by dissolving PCL in acetic acid to prepare a $15 \mathrm{wt} . \%$ solution. This solution was coated on the exterior of a $6 \mathrm{~mm}$ diameter PTFE rod, which served as the mould controlling the inside diameter of the tubular scaffold. Additional layers of coating were added to control the wall thickness within $0.20 \pm 0.02 \mathrm{~mm}$. To reinforce the scaffold, PET yarns were knitted into a $6 \mathrm{~mm}$ diameter single jersey-knitted structure using a 25-needle circular weft-knitting machine, which was specially designed and built in our laboratory. Three different densities (low, medium, and high) of knitted fabric were produced according to the codes in Table 2.

The PCL solution was coated on both sides of the tubular knitted structure prior to mounting on the $6 \mathrm{~mm}$ diameter PTFE rod. After coating, the PCL composite scaffolds were cooled and held at $-10^{\circ} \mathrm{C}$ for $12 \mathrm{~h}$. Afterwards, the scaffolds were freeze-dried at $-60^{\circ} \mathrm{C}$ under less than $10 \mathrm{~Pa}$ pressure for $5 \mathrm{~h}$ using an FD-1A-50 freeze drying machine. The scaffolds were subsequently immersed in a series of three distilled water baths, with each immersion lasting for $12 \mathrm{~h}$, to remove residual solvents, as shown in Figure 1.

\subsection{Characterisation Techniques}

2.3.1. Yarn Characterisation. Tensile measurements were performed on a YG061 electronic single yarn strength tester with an initial gauge length of $300 \mathrm{~mm}$ according to the Chinese standard GB/T 3916-1997.

2.3.2. Optical Microscopic Studies. The knitted structure was assessed using a Stereo Microscope PXS8-T (Nikon Co., Ltd.). Photos were taken by inserting glass (diameter of $6 \mathrm{~mm}$ ) into the weft-knitted tubular fabric under $2 \mathrm{x}$ magnification.

2.3.3. Geometrical Characteristic. Geometrical characteristics were determined according to ISO Standard 7198:1998 [23]. Results were averaged from 10 replicates.

Weight and Thickness. The weight and the thickness of the tubular fabrics and prototype vascular grafts were mea- sured using electronic measuring devices. Six-centimeterlong samples were prepared and weights (g) per square meter were calculated from the following:

$$
\text { Weight of sample }\left(\frac{\mathrm{g}}{\mathrm{m}^{2}}\right)=\frac{\text { weight of sample }(\mathrm{g})}{\pi d L},
$$

where $d$ is the diameter of the sample $(\mathrm{m})$ and $L$ is the length of the sample $(\mathrm{m})$.

PCL Content. The PCL content of each sample was calculated using the following:

$$
\begin{aligned}
& \text { PCL content }(\%) \\
& =\text { (weight of the composite samples } \\
& \quad \text { - weight of pure fabric) } \\
& \quad \times \text { (weight of the composite samples })^{-1} * 100 \text {. }
\end{aligned}
$$

Porosity Determination. The porosity $(P \%)$ of each sample was calculated from (3). The porosities of the PET weft-knitted tubular fabric and pure PCL prototype vascular graft were directly calculated from their densities:

$$
P(\%)=\left[1-\left(\frac{M}{100 h d}\right)\right] * 100
$$

where $M$ is the mass per unit area of the sample $\left(\mathrm{g} / \mathrm{m}^{2}\right), h$ is the sample thickness $(\mathrm{mm})$, and $d$ is the density of the material $\left(\mathrm{g} / \mathrm{cm}^{3}\right)$.

The porosities of the composite prototype vascular grafts were calculated from the density of composite using the following:

$$
\mathrm{DC}=(\mathrm{CPET} * \mathrm{DPET})+(\mathrm{CPCL} * \mathrm{DPCL}),
$$

where DC is the density of composite prototype vascular grafts $\left(\mathrm{g} / \mathrm{cm}^{3}\right)$, DPET is the density of the PET $\left(\mathrm{g} / \mathrm{cm}^{3}\right)$, DPCL is the density of the PCL $\left(\mathrm{g} / \mathrm{cm}^{3}\right)$, CPET is the content of PET in the composite (\%), and CPCL is the content of PCL in the composite (\%).

2.3.4. Scanning Electron Microscopy (SEM). SEM experiments were performed to analyse the surface morphologies and cross sections of the PCL vascular grafts reinforced with weft-knitted tube fabric. A Jeol JSM-5600LV instrument was used. Before examination, samples were gold-sputtered under nitrogen at an excitation voltage of $15 \mathrm{kv}$.

2.3.5. Tensile Strength. The tensile strength of the samples (along the longitudinal and radial directions) was tested using a YG-B026H universal mechanical tester (Wenzhou Darong Textile Instrument Co., Ltd., China) according to ISO Standard 7198: 1998 [23] under a strain rate of $50 \mathrm{~mm} / \mathrm{min}$. Results were averaged from five replicates.

2.3.6. Water Permeability. A water reservoir was connected to a polyethylene tube. The $2.65 \mathrm{~cm}$ long graft (surface area of 


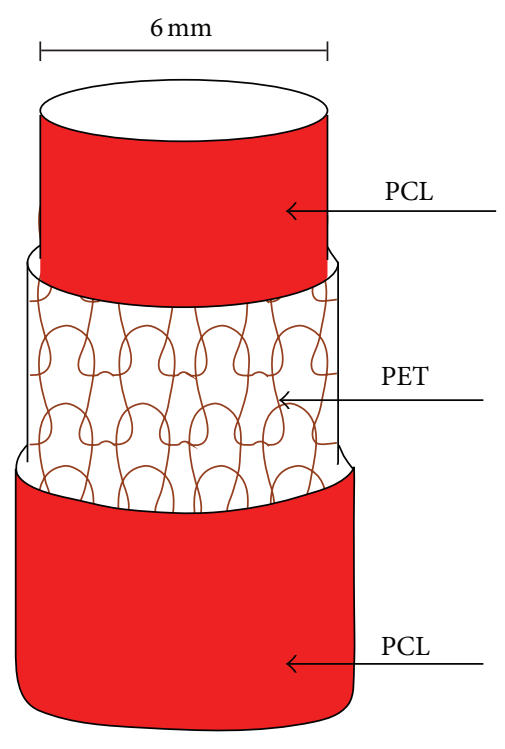

(a)

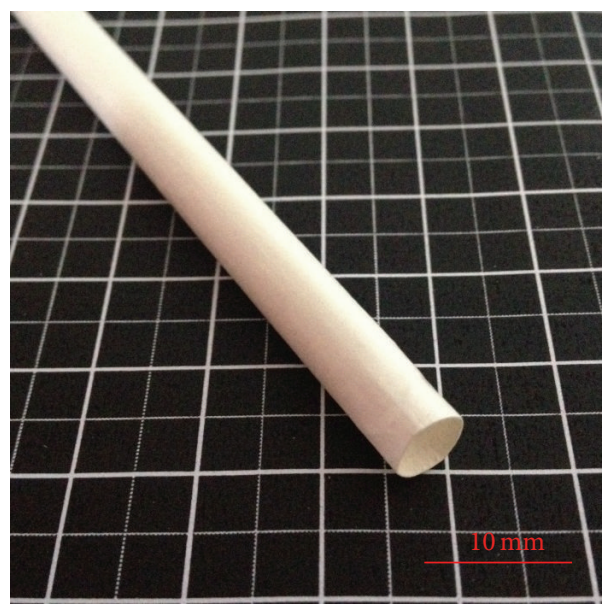

(b)

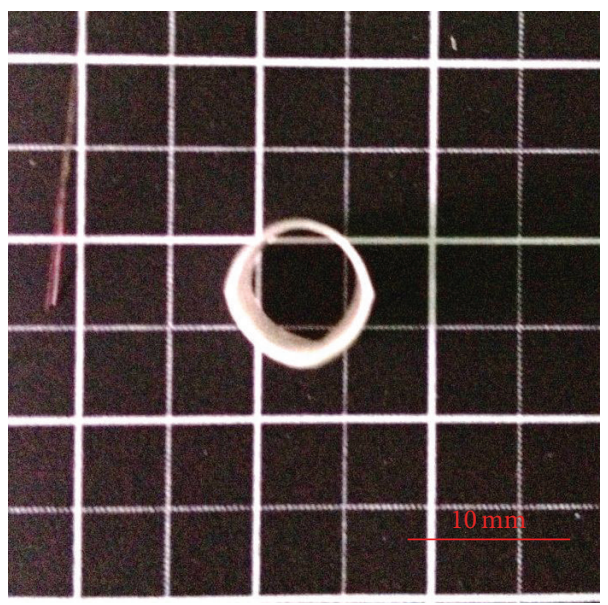

(c)

FIGURE 1: (a) Schematic representation of new prototype vascular graft. (b) and (c) Optical images of new prototype vascular grafts.

TABLE 1: Properties of polyester yarns.

\begin{tabular}{lcccc}
\hline Yarn code $^{*}$ & Yarn count (diner) & Tenacity $(\mathrm{cN} / \mathrm{dtex})$ & Elongation at break $(\%)$ & Density $\left(\mathrm{g} / \mathrm{cm}^{3}\right)$ \\
\hline Mono & $30 \mathrm{D}$ & $3.61 \pm 0.12$ & $16.36 \pm 2.35$ & 1.39 \\
Multi & $30 \mathrm{D} / 22 \mathrm{f}$ & $3.08 \pm 0.08$ & $94.40 \pm 16.78$ & 1.39 \\
\hline
\end{tabular}

${ }^{*}$ Mono: monofilament; Multi: multifilament.

$1 \mathrm{~cm}^{2}$ ) was connected to two conductors, and the group was connected to the device. The water reservoir was placed in a location elevated by about $165 \mathrm{~cm}$. The pressure transducer was used to verify that the distal end of the graft was constantly exposed to $120 \mathrm{mmHg}$ pressure. The water $(\mathrm{mL})$ permeating through the wall of the grafts $\left(\mathrm{cm}^{2}\right)$ over time (min) was collected in a graduated cylinder to calculate the water permeability in $\mathrm{mL} / \mathrm{cm}^{2} \mathrm{~min}$. Water permeability can be expressed using (5) according to ISO Standard 7198: 1998:

$$
W=\frac{Q}{A} \text {. }
$$

2.3.7. Radial Compression. Radial compression was tested using a YG061 radial compressive apparatus (Laizhou Electronic Instrument Co., Ltd., China). The testing method for the elastic recovery was based on the principles found in [24]. The strain rate was $10 \mathrm{~mm} / \mathrm{min}$, and the sample pressure was $50 \%$ of their diameter. Results were averaged from three replicates. The elastic recovery of each sample was calculated from (6) according to the curve in Figure 2:

$$
E \%=\left[\frac{\left(E_{1}+E_{2}\right)}{E_{T}}\right] * 100
$$


TABLE 2: Fabric densities of PET weft-knitted tube fabrics.

\begin{tabular}{lcccccc}
\hline NO & 1 & 2 & 3 & 4 & 5 & 6 \\
\hline Sample code & Mono(1) & Mono(2) & Mono(3) & Multi(1) & Multi(2) & Multi(3) \\
The density & High & Medium & Low & High & Medium & Low \\
\hline
\end{tabular}

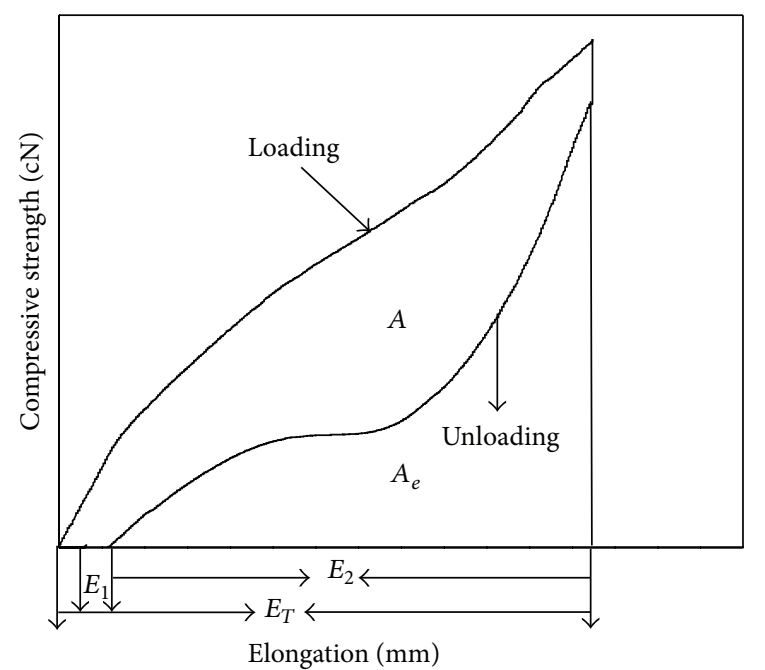

FIGURE 2: Calculation of the elastic recovery of prototype vascular grafts.

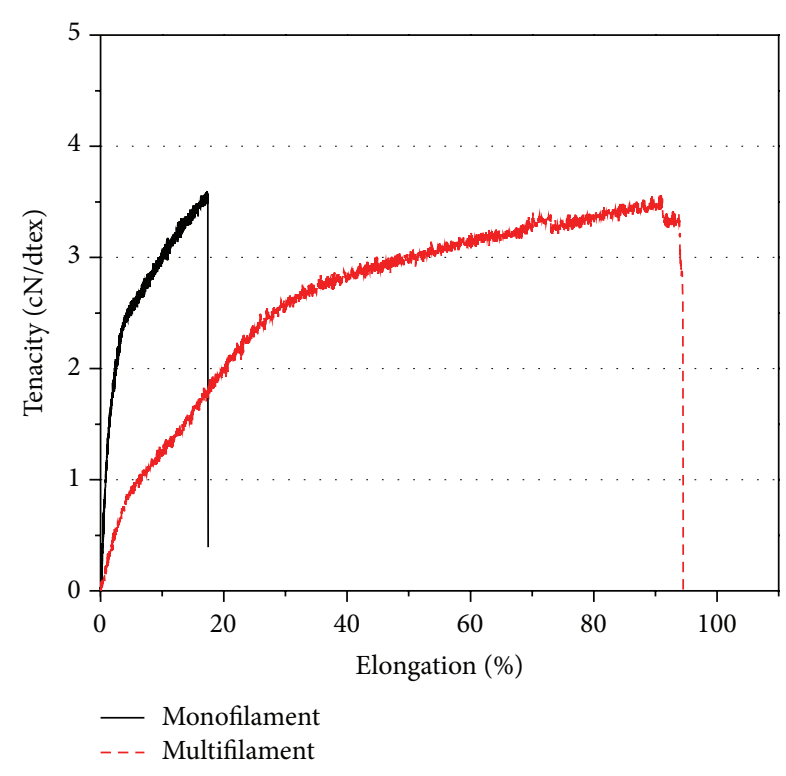

FIGURE 3: Elongation-strength curves of polyester yarns.

2.3.8. Suture Retention Strength. The suture retention strength was tested using a YG-B026H universal textile strength tester (Wenzhou Darong Textile Instrument Co., Ltd., China) according to ISO Standard 7198: 1998 [24]. The strain rate was $50 \mathrm{~mm} / \mathrm{min}$. Samples were tested in four positions and results were averaged from three replicates. The suture retention strength was calculated in terms of $\mathrm{N}$ and grams, similar to [25].

\section{Results and Discussion}

3.1. Yarn Tensile Strength. Figure 3 shows the tensile measurement results. The monofilament yarn showed slightly higher tenacity than the multifilament yarn, which is attributed to differences in the fibre composition of each sample. Multifilament yarns are known to be significantly less stiff and show lower compressive strengths than monofilament yarns [26].

3.2. PET Tubes Fabric. Figure 4 shows optical microscopic images of the PET tube fabrics. The images illustrate that loop cohesion and the cover factor are significantly better in the multifilament yarns (Figures 4(d), 4(e), and 4(f)) than in the monofilament yarns (Figures 4(a), 4(b), and 4(c)) because of the higher friction coefficient of the former compared with that of the latter. In addition, the loop density of the weftknitted tube fabric can be observed: Figures 4(a) and 4(d) show high densities, whilst Figures 4(c) and 4(f) show low densities.

Table 3 shows the loop densities and lengths of the different structures of the weft-knitted tube fabrics. Mono(1) and Multi(1) have higher densities than Mono(3) and Multi(3), respectively.

3.3. Geometrical Characteristic of Composite Prototype Vascular Grafts. Table 4 shows the geometric characteristic of the new composite prototype vascular graft. Fabric density generally affected the geometrical properties of the PCL composite vascular grafts. When the PCL (\%) was kept constant, increasing the fabric density increased the weight $\left(\mathrm{g} / \mathrm{m}^{2}\right)$ and thickness $(\mathrm{mm})$ of the resultant fabric. However the porosity of the PCL composite vascular grafts has no correlation effect with fabric densities, due to manual compositing, as seen in Table 4 . The new composite prototype vascular graft was thinner and lighter and showed better porosity than commercial ePTFE vascular grafts (Thin Wall). This result indicates that the new composite prototype vascular graft has promising potential in tissue engineering applications.

3.4. SEM. Microporous structures of PCL were fabricated using the freeze-drying method. Figure 5 shows SEM images of the surface and cross section of PCL reinforced with weft-knitted fabric as well as the surface section of the ePTFE vascular graft. PCL reinforced with weft-knitted fabric showed a pore size range of $10 \mu \mathrm{m}$ and higher, which indicates that this sample can be used for cell patterning and other clinical applications. The porosity of the new composite prototype was similar to that of ePTFE. Compared with the control ePTFE, however, the porosity of the pure PCL vascular graft yielded decreases in the mechanical properties of the sample. 


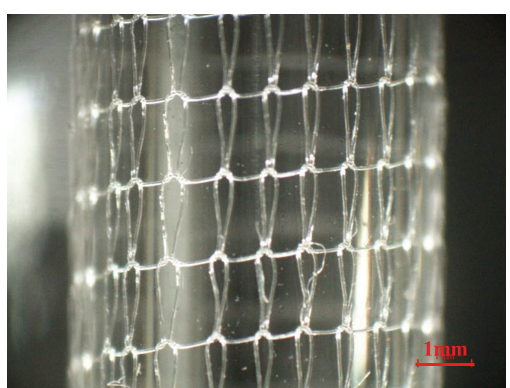

(a)

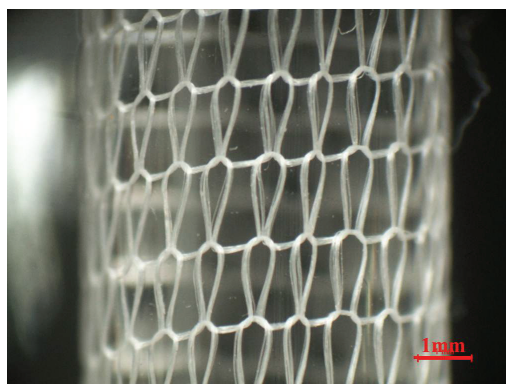

(d)

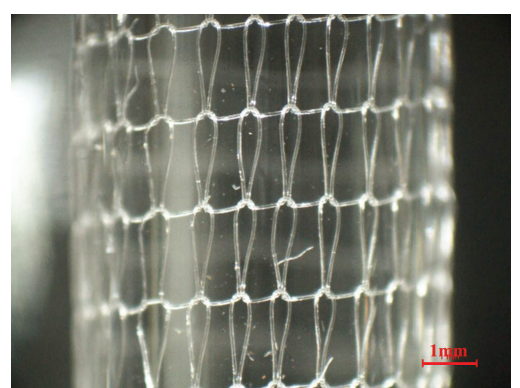

(b)

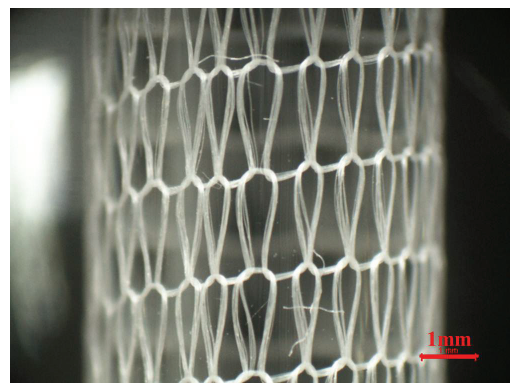

(e)

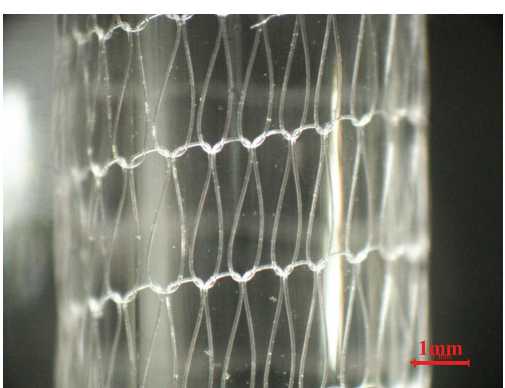

(c)

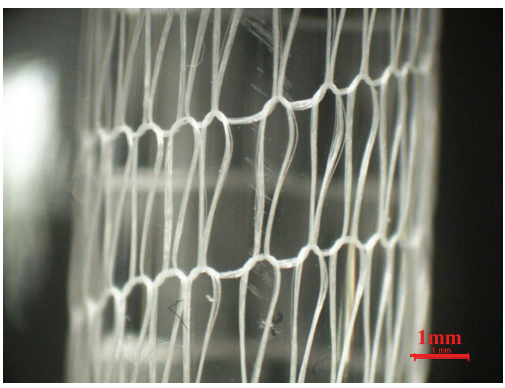

(f)

FIGURE 4: Optical microscopic images of the structures of weft-knitted fabric (X2): (a)-(c) monofilament yarns and (d)-(f) multifilament yarns.

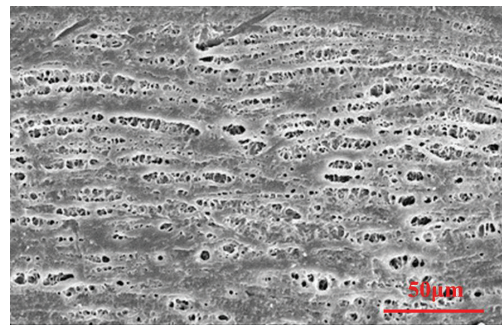

(a)

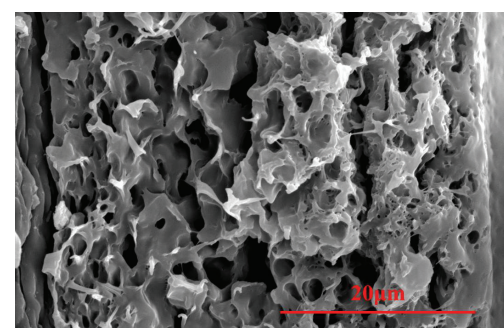

(b)

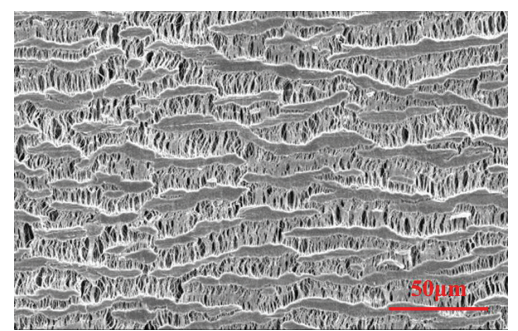

(c)

FIGURE 5: SEM images of microstructures of (a) surface section, (b) cross section of PCL composite prototype vascular graft, and (c) surface section of ePTFE vascular graft.

3.5. Tensile Strength of the PCL/PET Composite Prototype Vascular Grafts. Composite vascular grafts with the strength and stability of a knitted structure combine the elasticity and blood-proof properties of PCL. Table 5 shows the breaking strength and elongation at break in longitudinal and circumferential direction of the PCL composite vascular grafts with the different fabric density of the PET fabric. Variations in breaking strength and elongation at break are due to variations in fabric weight. The breaking strength in longitudinal direction is slightly lower than the ePTFE graft and the breaking strength in circumferential direction is better than the ePTFE graft. It is indicated that the composite PCL graft is of the potential clinical application for the breaking strength.

3.6. Water Permeability. The water permeability of the $\mathrm{PCL} / \mathrm{PET}$ composite prototype small-diameter vascular graft is zero (Table 6). In general, the water permeability of all of the samples was suitable for biomedical applications; according to some references, materials with a water permeability of less than $300 \mathrm{~mL} / \mathrm{cm}^{2}$ min may be employed in biomedical uses [23, 27]. Our results are similar to those observed in ePEFE vascular grafts produced by Vascutek. Compared with knitted and woven grafts produced by Sam Company, however, the water permeability of our graft was lower.

3.7. Radial Compressive Strength of the PCL/PET Composite Prototype Vascular Grafts. General analysis of Figure 6 shows that all of the samples have acceptable compressive strengths and elastic recoveries. Figure 6 and Table 7 indicate that fabric density exerts notable influences on the compressive strength and elastic recovery of the resultant graft. Amongst the fabric densities analysed, the vascular grafts produced with fabric densities of 2153.0 and $2086.5 \mathrm{loop} / 2500 \mathrm{~mm}^{2}$ 
TABLE 3: Density of PET monofilament and multifilament weft-knitted tube fabrics.

\begin{tabular}{lccccc}
\hline Number & Sample code & Wale $/(50 \mathrm{~mm})$ & Course $/(50 \mathrm{~mm})$ & Density/fabric $\left(2500 \mathrm{~mm}^{2}\right)$ & Length of loop $(\mathrm{mm})^{2}$ \\
\hline A & Mono(1) & 32.0 & 67.3 & 2153.0 & 1.6 \\
B & Mono(2) & 29.2 & 71.4 & 2086.5 & 1.8 \\
C & Mono(3) & 19.3 & 72.7 & 1901.5 & 2.6 \\
D & Multi(1) & 31.1 & 63.0 & 179.0 & 1.6 \\
E & Multi(2) & 25.1 & 68.6 & 1098.5 & 2.1 \\
F & Multi(3) & 15.8 & 69.6 & & 2.9 \\
\hline
\end{tabular}

TABLE 4: Characteristics of new composite prototype and ePTFE commercial vascular grafts.

\begin{tabular}{lccccc}
\hline Sample code & Fabric density $\left(\mathrm{loop} / 2500 \mathrm{~mm}^{2}\right)$ & Thickness $(\mathrm{mm})$ & Weight $\left(\mathrm{g} / \mathrm{m}^{2}\right)$ & PCL content $(\%)$ & Porosity $(\%)$ \\
\hline Mono(1) & 2153.0 & $0.275 \pm 0.02$ & $165.8 \pm 15.02$ & $90.81 \pm 0.87$ & $48.42 \pm 4.67$ \\
Mono(2) & 2086.5 & $0.260 \pm 0.01$ & $135.93 \pm 5.81$ & $90.69 \pm 0.67$ & $55.49 \pm 3.46$ \\
Mono(3) & 1401.5 & $0.167 \pm 0.02$ & $126.19 \pm 15.0$ & $90.14 \pm 0.72$ & $35.47 \pm 4.6$ \\
Multi(1) & 1959.0 & $0.235 \pm 0.02$ & $157.1 \pm 10.01$ & $90.71 \pm 0.57$ & $43.88 \pm 3.58$ \\
Multi(2) & 1723.2 & $0.193 \pm 0.02$ & $131.50 \pm 6.56$ & $90.66 \pm 0.66$ & $41.54 \pm 7.27$ \\
Multi(3) & 1098.5 & $0.187 \pm 0.01$ & $115.75 \pm 9.54$ & $90.03 \pm 0.81$ & $49.07 \pm 4.2$ \\
ePTFE & - & 0.458 & 338 & - & 45.63 \\
\hline
\end{tabular}

TABLE 5: Effect of fabric density on breaking strength and elongation at break.

\begin{tabular}{lcccc}
\hline \multirow{2}{*}{ Sample code } & \multicolumn{2}{c}{ Longitudinal } & \multicolumn{2}{c}{ Circumferential } \\
& Elongation at break (\%) & Breaking stress (MPa) & Elongation at break (\%) & Breaking stress (MPa) \\
\hline Mono(1) & $48.0 \pm 12.4$ & $12.7 \pm 0.2$ & $167.8 \pm 11.5$ & $7.1 \pm 0.1$ \\
Mono(2) & $58.9 \pm 16.8$ & $13.3 \pm 0.2$ & $160.4 \pm 15.5$ & $5.4 \pm 0.3$ \\
Mono(3) & $42.3 \pm 3.2$ & $19.2 \pm 0.1$ & $173.0 \pm 0.3$ & $9.8 \pm 0.1$ \\
Multi(1) & $48.3 \pm 8.4$ & $16.0 \pm 0.2$ & $128.5 \pm 9.0$ & $6.4 \pm 0.02$ \\
Multi(2) & $30.2 \pm 11.6$ & $12.9 \pm 0.4$ & $141.2 \pm 5.1$ & $4.8 \pm 0.3$ \\
Multi(3) & $43.3 \pm 11.8$ & $21.3 \pm 0.2$ & $167.1 \pm 1.2$ & $9.4 \pm 0.1$ \\
ePTFE & $59.6 \pm 4.4$ & $24.7 \pm 1.4$ & - & 4.67 \\
\hline
\end{tabular}

TABLE 6: Water performance of PCL/PET composite prototype and commercial ePTFE vascular grafts.

\begin{tabular}{|c|c|c|c|c|}
\hline Sample code & All our samples & Woven & Knitted & ePTFE \\
\hline Manufactures & Our work & & Vascutek & \\
\hline Water permeability $\left(\mathrm{mL} / \mathrm{cm}^{2} / \mathrm{min}\right)$ & 0 & 75.73 & 1126.3 & 0 \\
\hline
\end{tabular}

(monofilament and multifilament, resp.) showed better ability to resist mechanical compression and lower elastic recovery than the vascular grafts produced with fabric densities of 1401.5 and $1098.5 \mathrm{loop} / 2500 \mathrm{~mm}^{2}$ (monofilament and multifilament, resp.) when the PCL content (\%) was held constant. The vascular grafts produced with fabric densities 2153.0 and $2086.5 \mathrm{loop} / 2500 \mathrm{~mm}^{2}$ (monofilament and multifilament, resp.) showed the highest compressive strengths and are therefore the thickest samples fabricated. The elastic recoveries of these grafts, however, were relatively low because of the high density of the fabrics.

3.8. Effect of Fabric Density on Suture Retention Strength. Figure 7 shows that all of the samples have suture retention strengths within the range of $370-420 \mathrm{~g}$, which is acceptable according to [25]. However, these suture strengths are low compared with those of ePTFE from Vascutek (see
Table 8), an observation that may be attributed to differences in thickness between samples. To increase the suture retention strength, the thickness of the sample must be increased.

Figure 7 illustrates the effect of fabric density on the suture retention strength. The figure clearly shows that when the fabric density increases, the suture retention strength also increases. This result is attributed to the decrease in distance between the yarns, which leads to stronger suture connections.

\section{Conclusions}

In summary, a new composite prototype vascular graft was successfully fabricated. This vascular graft features excellent pore sizes, tensile strength, water permeability, elastic recovery, and suture retention strength. The graft shows promising 
TABLE 7: Compressive strength and elastic recovery of PCL/PET composite prototype vascular grafts.

\begin{tabular}{lccc}
\hline Sample code & Fabric density $\left(\mathrm{loop} / 2500 \mathrm{~mm}^{2}\right)$ & Compressive strength $(\mathrm{cN})$ & Elastic recovery $(\%)$ \\
\hline Mono(1) & 2153.0 & 96.01 & 93.54 \\
Mono(2) & 2086.5 & 94.25 & 94.16 \\
Mono(3) & 1401.5 & 90.51 & 98.64 \\
Multi(1) & 1959.0 & 120.9 & 92.19 \\
Multi(2) & 1723.2 & 114.7 & 94.87 \\
Multi(3) & 1098.5 & 105.3 & 95.90 \\
\hline
\end{tabular}

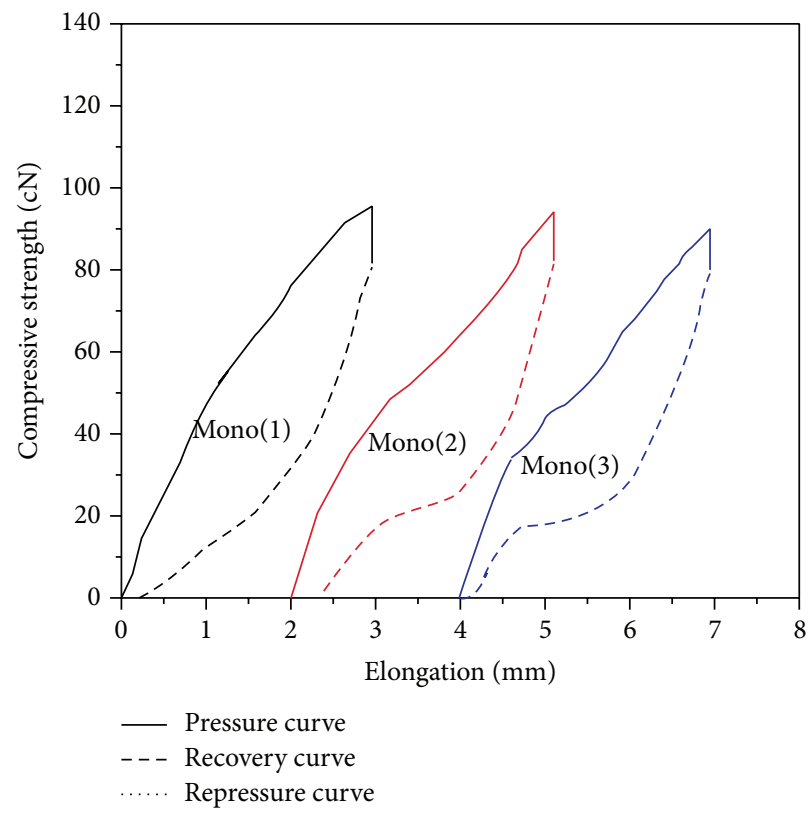

(a)

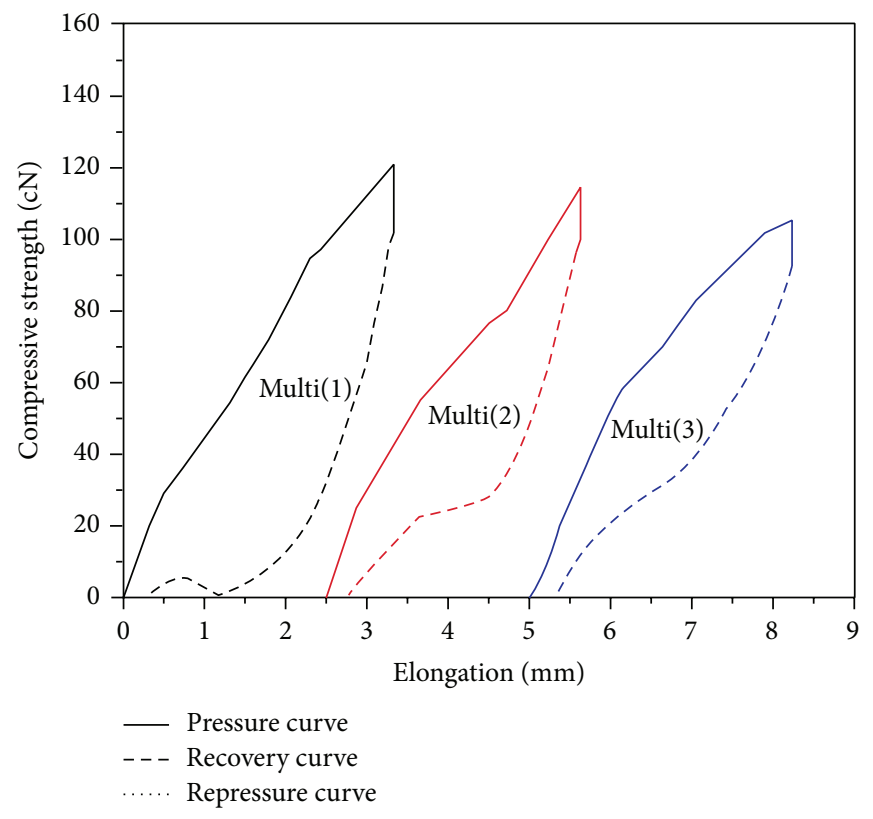

(b)

FIGURE 6: Elastic recovery curves of PCL/PET composite prototype vascular grafts: (a) monofilament and (b) multifilament.

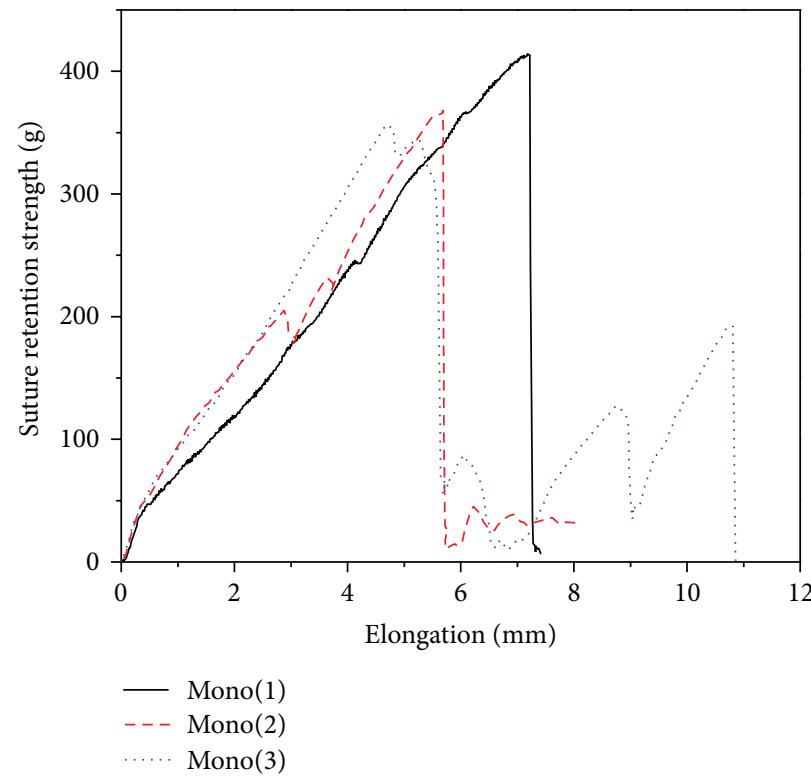

(a)

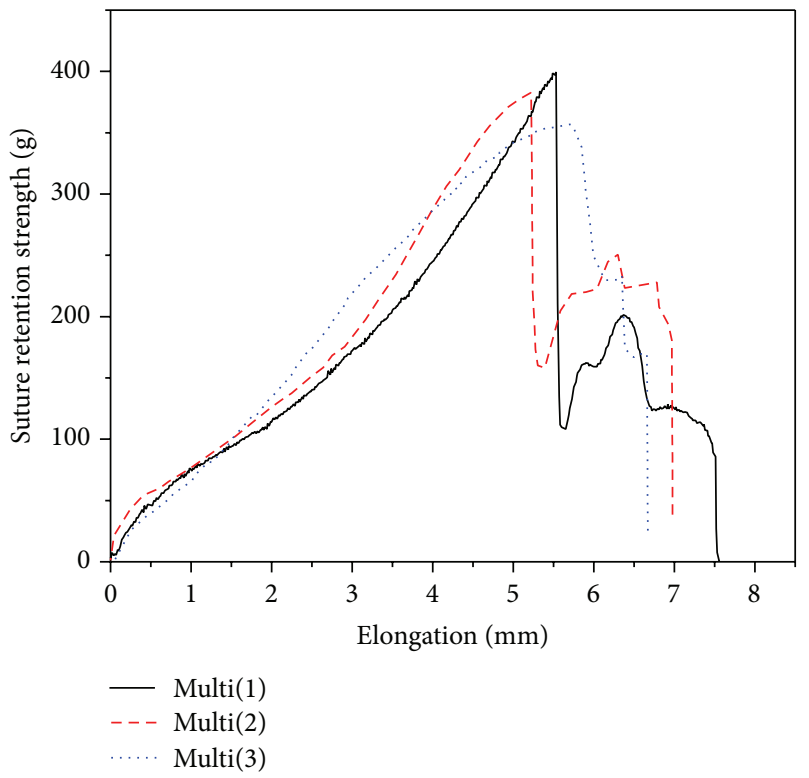

(b)

FIGURE 7: Suture retention strength curves of prototype vascular grafts with different fabric densities: (a) monofilament and (b) multifilament. 
TABLE 8: Suture strengths at break of PCL/PET composite prototype and ePTFE commercial vascular grafts.

\begin{tabular}{lccccccc}
\hline Sample code & Mono(1) & Mono(2) & Mono(3) & Multi(1) & Multi(2) & Multi(3) & ePTFE \\
\hline Suture strength at break (g) & $421.4 \pm 77.3$ & $412.3 \pm 113.6$ & $385.5 \pm 130.2$ & $402.1 \pm 67.4$ & $394.0 \pm 120.8$ & $371.0 \pm 120.8$ & $833.9 \pm 544.1$ \\
Suture strength at break (N) & 0.413 & 0.404 & 0.378 & 0.394 & 0.386 & 0.364 & 0.818 \\
\hline
\end{tabular}

potential for composite scaffold development in vascular graft applications and tissue engineering.

Fabric density showed significant effects on the mechanical properties of the graft. As the loop density was increased, increases in compressive strength and suture retention strength were observed; decreases in elastic recovery, however, were further noted. Meanwhile the mechanical properties are close to or better than the commercial ePTFE graft, which indicated that the composite PCL graft is promising in potential application for small-diameter vascular graft.

\section{Conflict of Interests}

The authors declare that there is no conflict of interests regarding the publication of this paper.

\section{Acknowledgments}

This work was supported by the Fundamental Research Funds for the Central Universities (Donghua University NS2013), the National Nature Science Foundation (31100682), the 111 Project "Biomedical Textile Materials Science and Technology" (B07024), and the Engineering Research Center of Technical Textiles Ministry of Education in China.

\section{References}

[1] D. P. Faxon, V. Fuster, P. Libby et al., "Atherosclerotic vascular disease conference. Writing group III: pathophysiology," Circulation, vol. 109, no. 21, pp. 2617-2625, 2004.

[2] S. Manju, C. V. Muraleedharan, A. Rajeev, A. Jayakrishnan, and R. Joseph, "Evaluation of alginate dialdehyde cross-linked gelatin hydrogel as a biodegradable sealant for polyester vascular graft," Journal of Biomedical Materials Research B: Applied Biomaterials, vol. 98, no. 1, pp. 139-149, 2011.

[3] R. Kowalewski, L. Zimnoch, M. Z. Wojtukiewicz, J. Glowinski, and S. Glowinski, "Expression of fibrinolysis activators and their inhibitor in neointima of polyester vascular grafts," Biomaterials, vol. 25, no. 28, pp. 5987-5993, 2004.

[4] P. Wong, S. Hopkins, D. Vincente, K. Williams, N. Macri, and R. Berguer, "Differences in neointima formation between impervious and porous polytetrafluoroethylene vascular patch material," Annals of Vascular Surgery, vol. 16, no. 4, pp. 407-412, 2002.

[5] G. J. Toes, K. W. van Muiswinkel, W. van Oeveren et al., "Superhydrophobic modification fails to improve the performance of small diameter expanded polytetrafluoroethylene vascular grafts," Biomaterials, vol. 23, no. 1, pp. 255-262, 2002.

[6] K. Hirabayashi, E. Saitoh, H. Ijima, T. Takenawa, M. Kodama, and M. Hori, "Influence of fibril length upon ePTFE graft healing and host modification of the implant," Journal of Biomedical Materials Research, vol. 26, no. 11, pp. 1433-1447, 1992.
[7] A. W. Clowes, T. R. Kirkman, and M. A. Reidy, "Mechanisms of arterial graft healing. Rapid transmural capillary ingrowth provides a source of intimal endothelium and smooth muscle in porous PTFE prostheses," The American Journal of Pathology, vol. 123, no. 2, pp. 220-230, 1986.

[8] M. A. Eliezer, M. M. Lydia, V. R. Virna, and F. R. Carlos, Application of Engineering Mechanics in Medicine, GED-University of Puerto Rico, 2005.

[9] R. A. White, ASAIO Transactions/American Society for Artificial Internal Organs, vol. 34, 1988.

[10] H. W. Weizsacker and J. G. Pinto, "Isotropy and anisotropy of the arterial wall," Journal of Biomechanics, vol. 21, no. 6, pp. 477$487,1988$.

[11] K. Hayashi, "Experimental approaches on measuring the mechanical properties and constitutive laws of arterial walls," Journal of Biomechanical Engineering, vol. 115, no. 4B, pp. 481488, 1993.

[12] U. Edlund, S. Dånmark, and A. Albertsson, "A strategy for the covalent functionalization of resorbable polymers with heparin and osteoinductive growth factor," Biomacromolecules, vol. 9, no. 3, pp. 901-905, 2008.

[13] Y. Wan, G. Feng, F. H. Shen, C. T. Laurencin, and X. Li, "Biphasic scaffold for annulus fibrosus tissue regeneration," Biomaterials, vol. 29, no. 6, pp. 643-652, 2008.

[14] K. W. Ng, H. N. Achuth, S. Moochhala, T. C. Lim, and D. W. Hutmacher, "In vivo evaluation of an ultra-thin polycaprolactone film as a wound dressing," Journal of Biomaterials Science, vol. 18, no. 7, pp. 925-938, 2007.

[15] X. Mo, H. J. Weber, and S. Ramakrishna, "PCL-PGLA composite tubular scaffold preparation and biocompatibility investigation," The International Journal of Artificial Organs, vol. 29, pp. 790-799, 2006.

[16] D. Y. Wong, S. J. Hollister, P. H. Krebsbach, and C. Nosrat, "Poly( $\varepsilon$-caprolactone) and poly (L-lactic-co-glycolic acid) degradable polymer sponges attenuate astrocyte response and lesion growth in acute traumatic brain injury," Tissue Engineering, vol. 13, no. 10, pp. 2515-2523, 2007.

[17] E. Pektok, B. Nottelet, J. Tille et al., "Degradation and healing characteristics of small-diameter poly( $\varepsilon$-caprolactone) vascular grafts in the rat systemic arterial circulation," Circulation, vol. 118 , no. 24, pp. 2563-2570, 2008.

[18] I. Olabarrieta, D. Forsström, U. W. Gedde, and M. S. Hedenqvist, "Transport properties of chitosan and whey blended with poly( $\boldsymbol{\varepsilon}$-caprolactone) assessed by standard permeability measurements and microcalorimetry," Polymer, vol. 42, no. 9, pp. 4401-4408, 2001.

[19] P. Xiang, M. Li, C. Zhang, D. Chen, and Z. Zhou, "Cytocompatibility of electrospun nanofiber tubular scaffolds for small diameter tissue engineering blood vessels," International Journal of Biological Macromolecules, vol. 49, no. 3, pp. 281-288, 2011.

[20] M.-C. Serrano, R. Pagani, M. Vallet-Regi, J. Pena, J.-V. Comas, and M.-T. Portoles, "Nitric oxide production by endothelial 
cells derived from blood progenitors cultured on $\mathrm{NaOH}-$ treated polycaprolactone films: a biofunctionality study," Acta Biomaterialia, vol. 5, no. 6, pp. 2045-2053, 2009.

[21] C. C. Chu and J. Rawlinson, "Mathematical modeling of water permeability of surgical fabrics for vascular use," Journal of Biomedical Materials Research, vol. 28, no. 4, pp. 441-448, 1994.

[22] F. Dieval, N. Chakfé, L. Wang et al., "Mechanisms of rupture of knitted polyester vascular prostheses: an in vitro analysis of virgin prostheses," European Journal of Vascular and Endovascular Surgery, vol. 26, no. 4, pp. 429-436, 2003.

[23] ISO7198, Cardiovascular implants-Tubularvascular prostheses.

[24] G. H. Liu, H. Hu, P. H. Zhang, and W. Z. Wang, "Radial compressive properties of the biodegradable braided regeneration tubes for peripheral nerve repair," Journal of Industrial Textiles, vol. 36, no. 1, pp. 35-46, 2006.

[25] Y. Mine, H. Mitsui, Y. Oshima, Y. Noishiki, M. Nakai, and S. Sano, "Suture retention strength of expanded polytetraf luoroethylene (ePTFE) graft," Acta Medica Okayama, vol. 64, no. 2, pp. 121-128, 2010.

[26] K. L. Hatch, Textile Science, West Publishing, 1993.

[27] M. W. King, Y. Marois, R. Guidoin et al., "Evaluating the Dialine vascular prosthesis knitted from an alternative source of polyester yarns," Journal of Biomedical Materials Research, vol. 29, no. 5, pp. 595-610, 1995. 

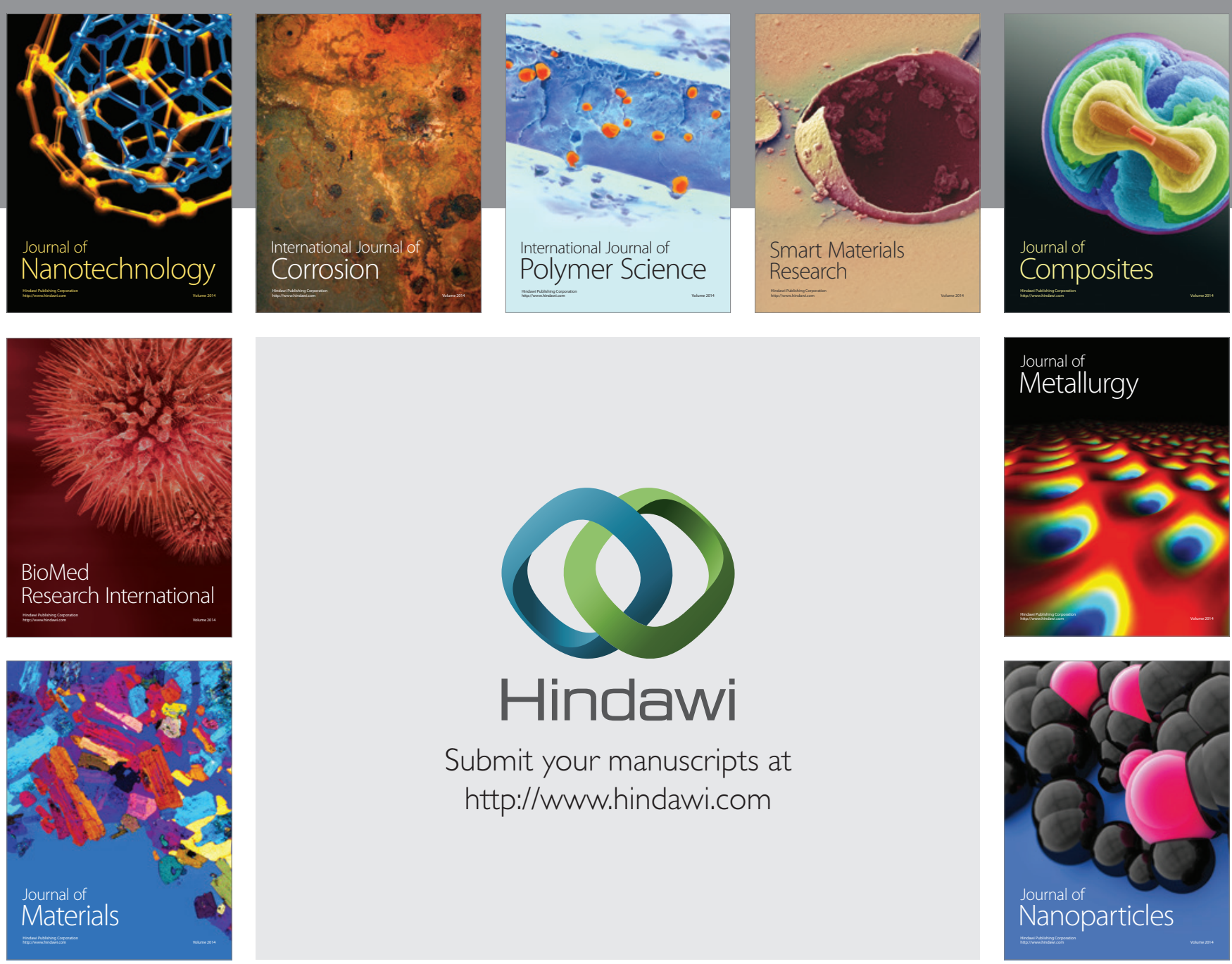

Submit your manuscripts at http://www.hindawi.com
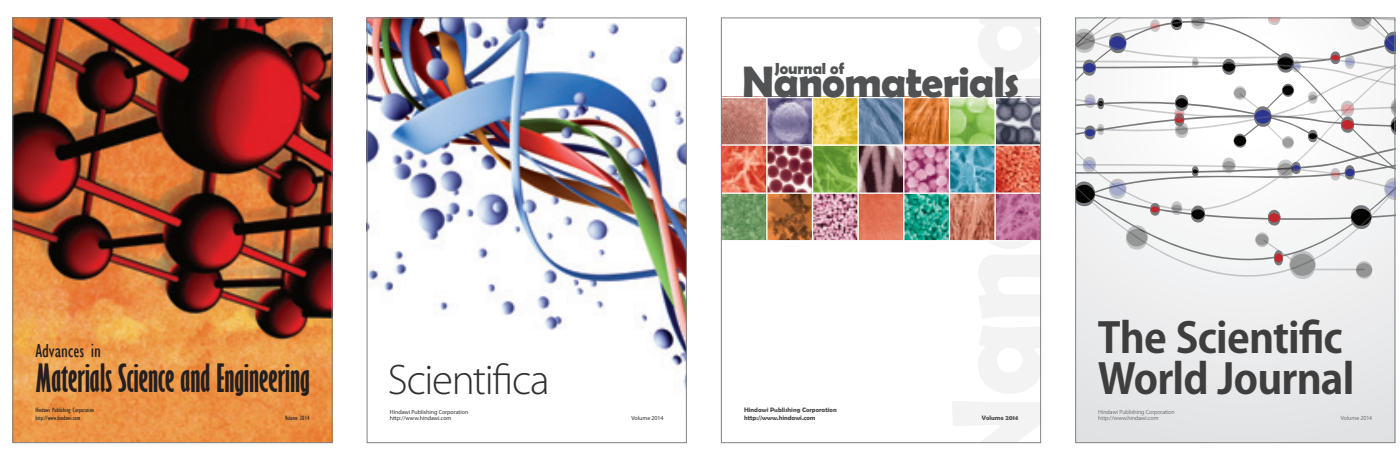

\section{The Scientific World Journal}
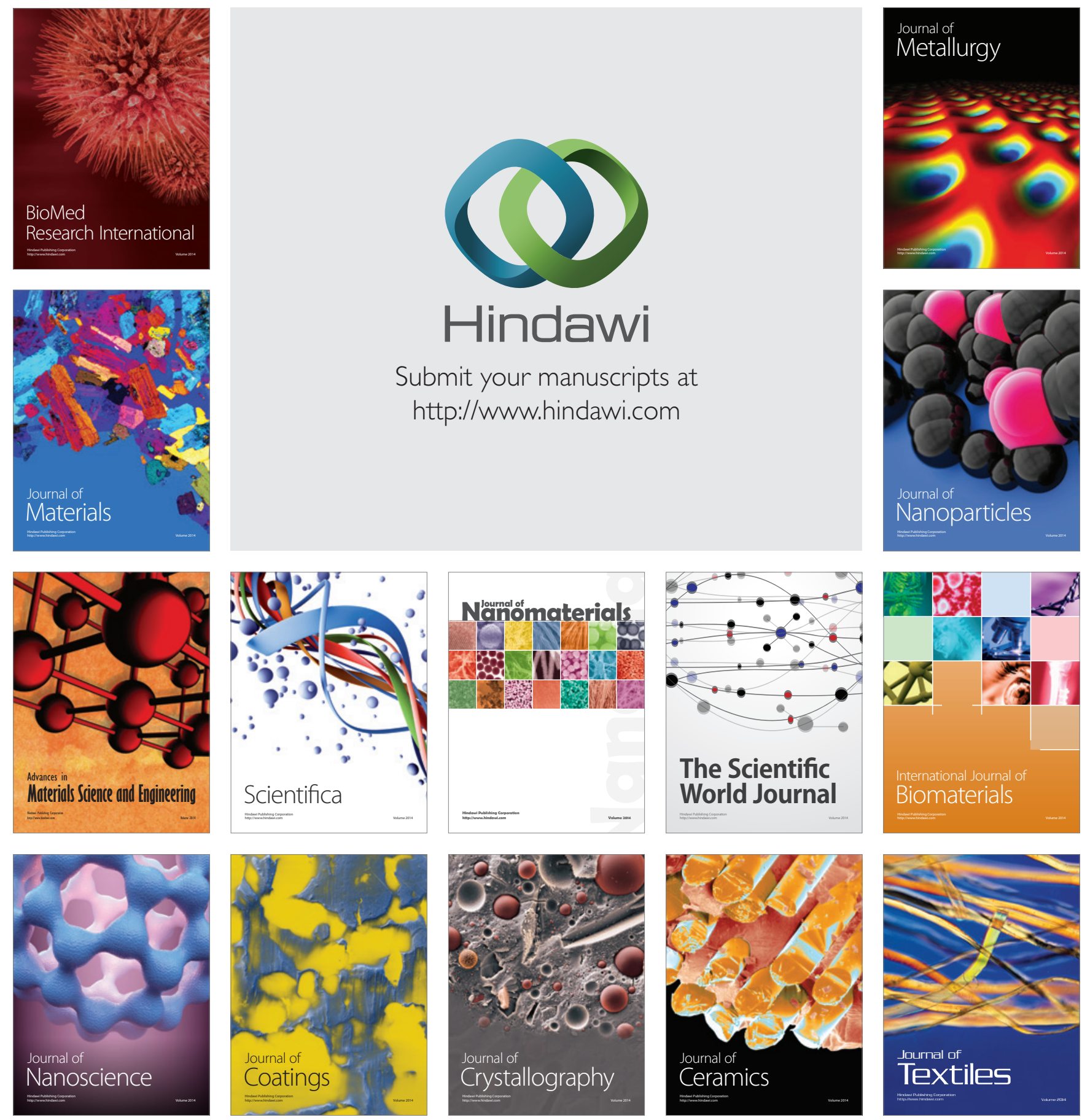